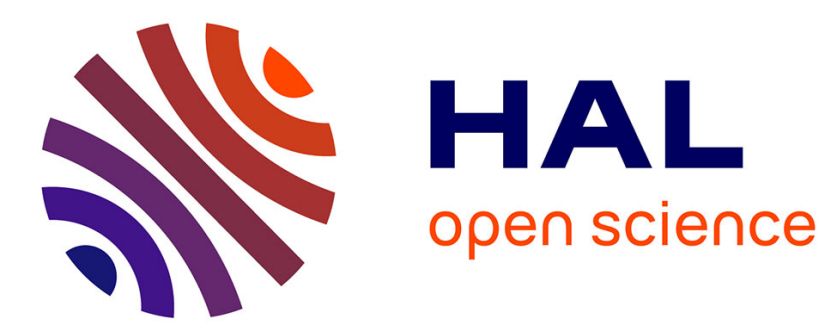

\title{
The role of boundary conditions in a simple model of incipient vortex breakdown
}

François Gallaire, Jean-Marc Chomaz

\section{To cite this version:}

François Gallaire, Jean-Marc Chomaz. The role of boundary conditions in a simple model of incipient vortex breakdown. Physics of Fluids, 2004, 16 (2), pp.274-286. 10.1063/1.1630326 . hal-01024945

\section{HAL Id: hal-01024945 \\ https://hal-polytechnique.archives-ouvertes.fr/hal-01024945}

Submitted on $30 \mathrm{Jul} 2014$

HAL is a multi-disciplinary open access archive for the deposit and dissemination of scientific research documents, whether they are published or not. The documents may come from teaching and research institutions in France or abroad, or from public or private research centers.
L'archive ouverte pluridisciplinaire HAL, est destinée au dépôt et à la diffusion de documents scientifiques de niveau recherche, publiés ou non, émanant des établissements d'enseignement et de recherche français ou étrangers, des laboratoires publics ou privés. 


\title{
AIP | Physics of Fluids
}

\section{The role of boundary conditions in a simple model of incipient vortex breakdown}

\author{
F. Gallaire and J.-M. Chomaz
}

Citation: Physics of Fluids (1994-present) 16, 274 (2004); doi: 10.1063/1.1630326

View online: http://dx.doi.org/10.1063/1.1630326

View Table of Contents: http://scitation.aip.org/content/aip/journal/pof2/16/2?ver=pdfcov

Published by the AIP Publishing

\section{Articles you may be interested in}

Evolution of a hairpin vortex in a shear-thinning fluid governed by a power-law model

Phys. Fluids 25, 101703 (2013); 10.1063/1.4824457

Study of instabilities and quasi-two-dimensional turbulence in volumetrically heated magnetohydrodynamic flows in a vertical rectangular duct

Phys. Fluids 25, 024102 (2013); 10.1063/1.4791605

Study of instabilities and transitions for a family of quasi-two-dimensional magnetohydrodynamic flows based on a parametrical model

Phys. Fluids 24, 024101 (2012); 10.1063/1.3680864

Control of axisymmetric vortex breakdown in a constricted pipe: Nonlinear steady states and weakly nonlinear asymptotic expansions

Phys. Fluids 23, 084102 (2011); 10.1063/1.3610380

On the development of three-dimensional vortex breakdown in cylindrical regions

Phys. Fluids 18, 084105 (2006); 10.1063/1.2338065

\section{AIP hamide}

Journal of Applied Physics is pleased to announce André Anders as its new Editor-in-Chief 


\title{
The role of boundary conditions in a simple model of incipient vortex breakdown
}

\author{
F. Gallaire ${ }^{\text {a) }}$ and J.-M. Chomaz \\ Laboratoire d'Hydrodynamique (LadHyX), CNRS, École Polytechnique, 91128 Palaiseau Cedex, France
}

(Received 22 October 2002; accepted 8 October 2003; published online 15 December 2003)

\begin{abstract}
We consider incipient vortex breakdown and describe how infinitesimal perturbations may destabilize a columnar swirling jet. The framework is axisymmetric and inviscid following Wang and Rusak's [J. Fluid Mech. 340, 177 (1997)] analysis. The goal of the present study is to relate the local properties of swirling flows in infinite pipes to their global stability properties in pipes of finite length. A spatial linear stability analysis is pursued which gives a complementary point of view to the subcritical/supercritical concept introduced by Benjamin [J. Fluid Mech. 14, 593 (1962)]. In contrast to supercritical flows which exhibit two neutral spatial branches traveling downstream and two counterpropagating evanescent spatial branches, subcritical flows exhibit a frequency range where all spatial branches are neutral, three of which travel downstream and one upstream. By using global energy budget arguments and monitoring how the upstream wave is reflected into the downstream waves and conversely, the inlet and outlet conditions are shown to drive the instability in the limit of long but finite pipes. Various inlet and outlet conditions are proposed that stabilize or destabilize the flow, depending on their ability to supply energy. The analysis demonstrates therefore that the global instability accounting for incipient vortex breakdown in Wang and Rusak's model may arise from the combination of a locally neutral flow and suitable inlet and outlet conditions.

(C) 2004 American Institute of Physics. [DOI: 10.1063/1.1630326]
\end{abstract}

\section{INTRODUCTION}

Vortex breakdown is an abrupt change in the vortex core structure with the appearance of a stagnation point close to the vortex axis. This widespread phenomenon commonly occurs in industrial or atmospheric conditions. In the laboratory, it is preferentially studied in vortex tubes, where it is seen in many cases to give rise to an axisymmetric structure. In such a configuration, the swirling flow is open and it should be supplemented with suitable inlet and outlet conditions. Vortex breakdown often involves hysteresis and has been recognized as a strongly nonlinear phenomenon. Nevertheless, a long series of studies starting with Squire ${ }^{1}$ and Benjamin $^{2}$ have shown that some physical insight may be gained by considering the dynamics of infinitesimal disturbances. When the swirl is increased slowly enough, transition to vortex breakdown is triggered by the loss of stability of the columnar state. The determination of this threshold for incipient vortex breakdown remains a formidable task since, in open flows, it is important to consider the evolution of perturbations not only in time but also in space $^{3}$ and to take into account the advection of the perturbations by the base flow. This has more recently led to examine the role of absolute instabilities, i.e., instabilities that grow in place, in the occurrence of self-sustained oscillations or so-called global modes (see Huerre and Rossi ${ }^{4}$ ). In particular, Chomaz and Couairon $^{5}$ have analyzed the combined effects of the local nature of the instability and the boundary conditions in systems of finite length. Absolute instability is not the only ex-

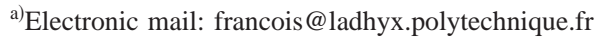

planation for global instability since any kind of feedback mechanism may be destabilizing. In the present paper we identify another example of global instability that results from the combination of a flow that can propagate energy in both directions without amplification and of inlet and outlet conditions that serve as energy providers. A similar behavior has recently been identified in fluid conveying pipes by Doaré and de Langre. ${ }^{6}$

In the context of vortex breakdown in swirling jets, Squire ${ }^{1}$ introduced the concept of critical state, by which he meant the amount of swirl necessary to sustain a standing ${ }^{7}$ wave of infinite wavelength. He also added that as the swirl is increased above this critical value, standing waves of finite wavelength will exist and propagate disturbances upstream. In other words, Squire's basic idea is the following: if standing waves exist on a vortex flow, then disturbances which are present downstream will propagate upstream along the vortex thereby causing breakdown even if they are not amplified.

Benjamin $^{2}$ introduced the concept of supercritical/ subcritical swirling flows. Vortices with axial flow are classified according to the propagating behavior of the nondispersive axisymmetric neutral Kelvin waves which they support in the low-wave-number limit. Such flows are said to be supercritical if they sustain only downstream-traveling waves and subcritical if they sustain both upstream and downstream traveling waves. This criterion is based on the sign of the phase velocity, meaning, for instance, that surfaces of constant phase cannot propagate upstream when the flow is supercritical. Benjamin ${ }^{2}$ then criticized Squire's suggestion: "a serious objection to Squire's theory is that the 
group velocity of his standing waves is in fact directed downstream, which means that the waves can only form in the rear of a disturbing agency and cannot spread upstream."

Benjamin's claim was then generally adopted, for instance, by Escudier: " "whilst the phase velocity of the waves considered by Squire might be directed upstream, their group velocity was directed downstream so that no energy could be transported upstream and waves could only form in the rear of a disturbing agency." This has led to many attempts to generalize the concept of supercritical/subcritical flows, as done by Tsai and Widnall ${ }^{9}$ through an ad hoc group velocity criterion. More recently Delbende, Chomaz, and Huerre, ${ }^{10}$ Olendraru, Sellier, and Huerre, ${ }^{11}$ and Loiseleux, Chomaz, and Huerre ${ }^{12}$ among others have focused on the absolute/ convective classification of instabilities, which is nothing but the natural generalization of the supercritical/subcritical classification to the case of instability waves.

Only Leibovich ${ }^{13}$ points out that phase and group velocities coincide in the low-wave-number regime where waves become nondispersive and neutral. He moderates Benjamin's objection to Squire: "Benjamin criticized this conception, pointing out that the group velocity at criticality is positive in the downstream direction, so that in a columnar flow, disturbances cannot spread upstream from a disturbance source. Had Squire described the processes in an evolving vortex core, along the lines above, Benjamin's objection would not have seemed compelling."

All these linear analyses ${ }^{1,2,9-12}$ are local ${ }^{4,5}$ in the sense that the swirling flow is assumed columnar and extending both upstream and downstream to infinity without taking into account streamwise boundary conditions. Wang and Rusak ${ }^{14}$ choose instead to consider the nonlinear steady states sustained by an inviscid swirling flow in a pipe of finite length for specific inlet and outlet boundary conditions. The following bifurcation sequence is then identified as the swirl parameter is increased. As the swirl reaches $\Omega_{0}$, a first saddlenode bifurcation is encountered which gives rise to a stable state identified by the authors as breakdown state, and an unstable state, referred to as accelerated (since the axial velocity is observed to increase on the centerline), while the columnar vortex remains stable. Above $\Omega_{0}$, the flow is then bistable and only finite amplitude perturbations may trigger vortex breakdown. Above a higher critical swirl value $\Omega_{1}$, the columnar vortex loses its stability, as demonstrated by Wang and Rusak, ${ }^{15,16}$ and vortex breakdown may then be reached by infinitesimal perturbations of the columnar flow. This loss of stability occurs slightly above the swirl value $\Omega_{B}$ of Squire $^{1}$ at which standing waves of infinite wavelength are sustained and neutral, the small difference vanishing in the long pipe limit. It should be emphasized that this instability of the columnar state is global in nature and takes place while the vortex remains locally neutral. The present paper seeks to unravel the apparent paradox between global instability and local neutrality. In particular, the respective role of the boundary conditions and the inertial waves living in the bulk of the flow is analyzed in terms of energy amplification and propagation. This amplification mechanism of infinitely small perturbations is essential to explain how vortex breakdown develops and this initial stage will be referred to as incipient vortex breakdown. The subtle balance explaining the existence and eventually the stability of the breakdown state is far more difficult to analyze physically and is out of the scope of the present paper (the interested reader is referred to Wang and Rusak ${ }^{14}$ or Keller, Egly, and Exley ${ }^{17}$ for different perspectives on this nonlinear issue).

We begin with the spatial stability analysis of the swirling jet profiles used in Wang and Rusak. ${ }^{15}$ This spatial study is complementary to the usual temporal point of view and it clearly brings out the propagation of waves in the flow. It allows one to precisely analyze the feedback loop that destabilizes the flow and it yields a necessary condition for global instability: the flow should be locally subcritical and thereby allow energy propagation in both directions for the global instability to set in. A lower bound is thereby established for the swirl number corresponding to the development of the global instability in the limit of long but finite pipes.

The energy budget demonstrates the crucial role of the inlet and outlet conditions; furthermore, it leads to the derivation of a sufficient condition for stability based only on the consideration of the inlet and outlet boundary conditions. Particular boundary conditions are proposed that fulfill this stability condition and are indeed seen to quench the global instability, whatever swirl setting is considered. In other words, the energy budget analysis provides a second necessary condition for instability, based solely on the boundary conditions. In the long but finite pipe limit, and in all presently studied combinations of boundary conditions, flows that fulfill both necessary conditions are indeed seen to be unstable.

The paper aims at providing a link between the local group-velocity criterion of Squire ${ }^{1}$ and the global picture of Wang and Rusak. ${ }^{14}$ In Wang and Rusak's ${ }^{15}$ spirit, most results are derived on a simple model of swirling flows: a column of fluid flowing at constant axial velocity and in solid body rotation. As shown below, this model has the considerable advantage of not only providing a fully explicit dispersion relation but also of decoupling the radial and axial dependence, thereby making the energy propagation analysis tractable. It is expected that the results will continue to hold for other flows provided the overall dynamics is projected on the first Kelvin branch and the coupling terms are neglected. Work is in progress along these lines.

The study is organized according to the following outline: the general formulation and equations are introduced in Sec. II. Section III details the classical temporal and less classical spatial stability analyses and it discusses Squire's and Benjamin's views. Section IV sets these local results in perspective when compared with Wang and Rusak's ${ }^{15}$ global mode picture. It is demonstrated that the upstream and downstream boundaries constitute the necessary energy provider to drive the instability. This clear identification of the origin of the instability allows us to propose different boundary conditions in Sec. V, that, depending on their ability to modify the energy budget, may stabilize or destabilize the columnar vortex. 


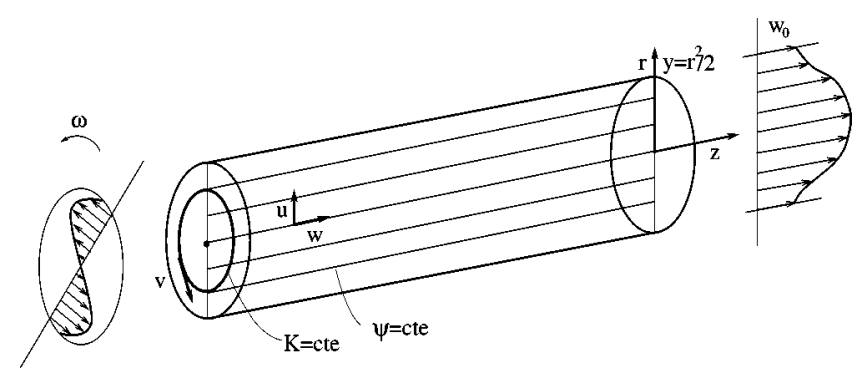

FIG. 1. Essential flow variables and base flow; axisymmetric stream surfaces $\psi(y, z, t)=$ const have been represented by their intersections with a meridional plane.

\section{FLOW CONFIGURATION AND FORMULATION}

\section{A. Flow configuration}

Consider axisymmetric swirling flow in a pipe of radius $R$ and length $l$. The viscosity is set to zero so that the flow is governed by the Euler equations. We use cylindrical coordinates $(r, \theta, z)$ and the velocity components $(u, v, w)$ correspond, respectively, to the radial, azimuthal, and axial velocity. The flow variables used in the study are sketched in Fig. 1.

The base flow consists of an axial flow of maximum velocity $w_{\max }$ superimposed on a rotation around the $z$ axis defined by the azimuthal velocity profile $v(r)$. The corresponding value of the axial vorticity measured on the axis equals $2 f$. Throughout the paper, we adopt the radius $R$ as length scale and $w_{\max }$ as velocity scale, so that the dimensionless columnar base flow reads

$$
u(r)=0 ; \quad v(r)=\frac{\sqrt{\Omega}}{2} v_{0}(r) ; w(r)=w_{0}(r),
$$

for $0 \leqslant r \leqslant 1,0 \leqslant z \leqslant L$, with $L=l / R$ being the nondimensional pipe length and $\Omega=4 f^{2} R^{2} / w_{\max }^{2}$ the swirl parameter. The axial velocity is assumed independent of $\Omega$ and the azimuthal velocity linearly dependent on $\sqrt{\Omega}$. The model under consideration is seen to involve two control parameters: the swirl $\Omega$ and the pipe aspect ratio $L$.

Depending on the velocity profiles $v_{0}(r)$ and $w_{0}(r)$ and on the swirl $\Omega$, the base flow, if considered infinite, will respond differently to external perturbations. It will either act as a waveguide for neutral waves or as an amplifying (damping) medium for unstable (stable) waves. Each of these behaviors is associated with a physical mechanism which sustains, amplifies, or damps the perturbations.

(1) Due to the restoring action of the Coriolis force, swirling flows present a remarkable radial "elasticity," which permits the propagation of longitudinal inertial waves, or so called Kelvin waves (see, for instance, $\operatorname{Saffman}^{18}$ ).

(2) The other force active in rotating flows, the centrifugal force, is able to destabilize the flow when certain requirements on the profiles are met: the famous Rayleigh ${ }^{19}$ criterion is a necessary and sufficient condition for centrifugal instability of the axisymmetric $m=0$ mode in the absence of axial flow. Howard and Gupta ${ }^{20}$ have generalized this criterion and derived a necessary condition for axisymmetric instability including the effects of advection. The criterion has been further extended to nonaxisymmetric perturbations by Ludwieg $^{21}$ and Leibovich and Stewartson ${ }^{22}$ but this is out of the scope of the present contribution.

(3) Independently of the specific azimuthal velocity profile, the Kelvin-Helmholtz mechanism may generate an unstable wave when strong shear is present in the axial velocity profile if the quantity $d w_{0}(r) / d r$ has a local extremum (see for details Batchelor and $\mathrm{Gill}^{23}$ ).

It is important to note that flows where none of these destabilizing agents (centrifugal or shear) are active, remain neutral if the extent of the flow is infinite in the axial direction. This is the case for the Rankine vortex with uniform axial flow, the Burgers vortex (i.e., a Lamb-Oseen vortex with uniform axial flow), or the Batchelor vortex. These flows have, however, been shown Wang and Rusak ${ }^{15}$ to become globally unstable when axially finite domains are considered, i.e., when suitable inlet and outlet conditions are added. Moreover, in these cases, Wang and Rusak ${ }^{14}$ and Rusak, Wang, and Whiting ${ }^{24}$ have demonstrated that the nonlinear saturated states resemble vortex breakdown bubbles. This is the primary motivation for restricting our study to profiles that remain locally neutral in an infinite domain.

\section{B. Squire-Long equations}

Let us introduce the circulation $K(r, z, t)=r v$, the stream function $\psi(r, z, t)$ such that $u=-\psi_{z} / r$ and $w=\psi_{r} / r$, the azimuthal vorticity $\eta$ and its reduced form $\chi=\eta / r$. Further defining a rescaled radial coordinate $y=r^{2} / 2$, the flow is governed by the unsteady Squire-Long equations (see Leibovich ${ }^{25}$ ):

$$
\begin{aligned}
& K_{t}+\psi_{y} K_{z}-\psi_{z} K_{y}=0, \\
& \chi_{t}+\psi_{y} \chi_{z}-\psi_{z} \chi_{y}=\frac{1}{4 y^{2}}\left(K^{2}\right)_{z}, \\
& \chi=-\left(\psi_{y y}+\psi_{z z} / 2 y\right) .
\end{aligned}
$$

Equation (2a) expresses the conservation of the azimuthal circulation $K$ in its transport along the flow. Similarly the left-hand side of the evolution equation (2b) accounts for the transport of azimuthal vorticity and its right-hand side for the tilting of axial vorticity into azimuthal vorticity. The stretching of the azimuthal vorticity is implicitly included through the use of the reduced variable $\chi$. As discussed in Szeri and Holmes, ${ }^{26}$ the problem is well-posed as soon as an inlet condition for $K$ and $\chi$ is specified as well as four boundary conditions for $\psi$ on each boundary of the domain. Since $y=0$ and $y=1 / 2$ are stream surfaces, the lateral boundary conditions are

$$
\begin{aligned}
& \psi(0, z, t)=0, \\
& \psi(1 / 2, z, t)=q,
\end{aligned}
$$

where $q$ denotes the nondimensional flow rate $q=\int_{0}^{1} r w_{0}(r) d r$. Three inlet conditions are added which effectively prescribe that the base flow be maintained at the inlet $z=0$ in the presence of perturbations

$$
\psi(y, 0, t)=\psi_{0}(y)
$$




$$
\begin{aligned}
& K(y, 0, t)=\sqrt{\Omega} K_{0}(y), \\
& \chi(y, 0, t)=\chi_{0}(y),
\end{aligned}
$$

where $\psi_{0}(y), K_{0}(y)$ are easily determined from the base flow (1) with the reference value $\psi_{0}(y=0)=0$ and where the inlet wall condition $\psi_{0}(1 / 2)=q$ compatible with relation (4) is imposed. As discussed in Wang and Rusak, ${ }^{14}$ the inlet vorticity is assumed to be fixed and equal to $\chi_{0}(y)=-\psi_{0 y y}(y)$. This choice is equivalent to imposing

$$
\psi_{z z}(0, y, t)=0
$$

at the inlet, letting the radial velocity $\psi_{z}(0, y, t)$ evolve freely but requiring the smooth cylindrical development of the flow leaving the inlet.

The outlet condition is the same as in Wang and Rusak: ${ }^{14}$

$$
\psi_{z}(y, L, t)=0,
$$

implying a vanishing radial velocity. Note that the columnar base flow $K(y, z, t)=\sqrt{\Omega} K_{0}(y), \quad \psi(y, z, t)=\psi_{0}(y)$, and $\chi(y, z, t)=\chi_{0}(y)$ is an exact solution of the inviscid nonlinear equations (3)-(9).

In contrast to the downstream boundary conditions, the choice of upstream boundary conditions has been a main source of concern in the literature. When an inviscid flow in a straight pipe of finite length is analyzed, Wang and Rusak ${ }^{14}$ have shown that the degree of freedom associated to the inlet radial velocity is of fundamental importance to sustain nonlinear vortex breakdown states. Note that these predicted states have been retrieved by the numerical simulations of Rusak, Wang, and Whiting. ${ }^{24}$ However, the bifurcation diagram described in the Introduction holds only when condition (8) is prescribed and any deviation from the specific choice of the inlet vorticity distribution in (8) leads to an imperfect transcritical bifurcation ${ }^{27}$ where the modified columnar state either disappears via a saddle-node bifurcation or transforms continuously into the accelerated state.

The boundary conditions to be applied when the fluid is made viscous are even more delicate. While Brown and Lopez $^{28}$ or Hafez et al. ${ }^{29}$ set the inlet radial velocity to zero, Kopecky and Torrance ${ }^{30}$ or Krause ${ }^{31}$ preferred to set the inlet azimuthal vorticity to zero. No matter what inlet condition was used, it was noticed that in this case, the vortex breakdown bubble tends to migrate upstream until it reaches (and crashes into) the boundary conditions. When the pipe geometry is modified by adding a constriction as in experimental devices, the influence of the choice of inlet boundary conditions becomes less crucial: on the one hand, Darmofal ${ }^{32}$ and Snyder and Spall ${ }^{33}$ prescribed a vanishing inlet radial velocity and successfully observed a breakdown bubble separated from the inlet, while, on the other hand, Beran and Culick ${ }^{34}$ and Lopez ${ }^{35}$ imposed instead a vanishing inlet vorticity and obtained similar results.

Snyder and Spall ${ }^{33}$ have furthermore shown that these simulations where the swirling flow is directly imposed at the inlet can satisfactorily reproduce the results of computations where the full geometry including the swirl generating guiding vanes is taken into account.

\section{Linearized equations}

Following Wang and Rusak, ${ }^{15}$ perturbations indicated by primed variables are superimposed on the base flow according to

$$
\begin{aligned}
& K(y, z, t)=\sqrt{\Omega} K_{0}(y)+K^{\prime}(y, z, t), \\
& \psi(y, z, t)=\psi_{0}(y)+\psi^{\prime}(y, z, t), \\
& \chi(y, z, t)=\chi_{0}(y)+\chi^{\prime}(y, z, t) .
\end{aligned}
$$

The Squire-Long equations $[(2 a)-(2 c)]$ are linearized around the columnar base flow. Introducing the modified Laplacian operator

$$
\widetilde{\Delta}=\frac{\partial^{2}}{\partial y^{2}}+\frac{1}{2 y} \frac{\partial^{2}}{\partial z^{2}},
$$

and eliminating $\chi^{\prime}$ through $\chi^{\prime}=-\widetilde{\Delta} \psi^{\prime}$ yields the twodimensional system

$$
\begin{aligned}
& {\left[\begin{array}{cc}
1 & 0 \\
0 & -\widetilde{\Delta}
\end{array}\right]\left(\begin{array}{l}
\dot{K}^{\prime} \\
\dot{\psi}^{\prime}
\end{array}\right)} \\
& =\left[\begin{array}{cc}
-\frac{\partial \psi_{0}}{\partial y} \frac{\partial}{\partial z} & \sqrt{\Omega} \frac{\partial K_{0}}{\partial y} \frac{\partial}{\partial z} \\
\sqrt{\Omega} \frac{K_{0}}{2 y^{2}} \frac{\partial}{\partial z} & -\frac{\partial \chi_{0}}{\partial y} \frac{\partial}{\partial z}-\frac{\partial \psi_{0}}{\partial y} \frac{\partial \widetilde{\Delta}}{\partial z}
\end{array}\right]\left(\begin{array}{l}
K^{\prime} \\
\psi^{\prime}
\end{array}\right),
\end{aligned}
$$

with the boundary conditions

$$
\begin{aligned}
& K^{\prime}(0, y, t)=0 ; \quad \psi^{\prime}(0, y, t)=0 ; \quad \psi_{z z}^{\prime}(0, y, t)=0 ; \\
& \psi_{z}^{\prime}(L, y, t)=0 ; \\
& \psi^{\prime}(z, 0, t)=0 ; \quad \psi^{\prime}(z, 1 / 2, t)=0 .
\end{aligned}
$$

In (11), the dot denotes the time derivative. When a global analysis is performed, all boundary conditions are taken into account and a solution is sought in terms of global modes of the form

$$
\begin{aligned}
& K^{\prime}(z, y, t)=\check{K}(z, y) e^{-i \omega t}, \\
& \psi^{\prime}(z, y, t)=\check{\psi}(z, y) e^{-i \omega t} .
\end{aligned}
$$

The global eigenfunctions depend both on the streamwise $z$ and radial $y$ coordinates whereas the long-time behavior is given by the sign of the imaginary part of $\omega$. The flow is stable if $\operatorname{Im}(\omega)<0$, neutral if $\operatorname{Im}(\omega)=0$, and unstable if $\operatorname{Im}(\omega)>0$.

When a local analysis is considered the inlet and outlet boundary conditions (12) are ignored and perturbations are decomposed into Fourier modes according to

$$
\begin{aligned}
K^{\prime}(z, y, t) & =\hat{K}(y) e^{i(k z-\omega t)}, \\
\psi^{\prime}(z, y, t) & =\hat{\psi}(y) e^{i(k z-\omega t)},
\end{aligned}
$$

where $k$ denotes the axial wave number. Only the radial boundary conditions (13) are conserved. The system (10) then reduces to the Kelvin wave equation $\left(\right.$ Benjamin $\left.^{2}\right)$ 


$$
\frac{d^{2} \hat{\psi}}{d y^{2}}-\left[\frac{k^{2}}{2 y}+\frac{d \chi_{0} / d y}{d \psi_{0} / d y-c}-\frac{\Omega K_{0} d K_{0} / d y}{2 y^{2}\left(d \psi_{0} / d y-c\right)^{2}}\right] \hat{\psi}=0,
$$

where $c=\omega / k$ is the phase velocity. This equation has been widely studied in the literature (see Leibovich ${ }^{13}$ for a review). In its stationary form, i.e., when $c$ is set to zero, it may be considered either as an eigenvalue problem in $\Omega$ with $k$ prescribed, or as an eigenvalue problem in $k$ with $\Omega$ prescribed. In its nonstationary form, $\Omega$ is considered as a parameter and if $k(\omega)$ is prescribed and assumed to be real, complex eigenfrequencies $\omega$ (eigenwavenumbers $k$ ) are looked for. The first of these approaches ( $k$ given real) is referred to as the local temporal approach since it examines the evolution in time of waves periodic in space, whereas the second ( $\omega$ given real) corresponds to local spatial stability theory. For definiteness, we consider the flow made up of a solid body rotation with uniform axial flow. One should keep in mind that the reasoning is valid for all neutrally stable profiles confined in pipes. In that case, $K_{0}(y)=y$, $\psi_{0}(y)=y$, and $\chi_{0}(y)=0$. Equation (11) becomes

$$
\left[\begin{array}{cc}
1 & 0 \\
0 & -\widetilde{\Delta}
\end{array}\right]\left(\begin{array}{l}
\dot{K}^{\prime} \\
\dot{\psi}^{\prime}
\end{array}\right)=\left[\begin{array}{cc}
-\frac{\partial}{\partial z} & \sqrt{\Omega} \frac{\partial}{\partial z} \\
\sqrt{\Omega} \frac{1}{2 y} \frac{\partial}{\partial z} & -\frac{\partial \widetilde{\Delta}}{\partial z}
\end{array}\right]\left(\begin{array}{l}
K^{\prime} \\
\psi^{\prime}
\end{array}\right),
$$

associated to boundary conditions (12) and (13), and Eq. (16) simplifies into

$$
\frac{d^{2} \hat{\psi}}{d y^{2}}-\left[\frac{k^{2}}{2 y}-\frac{\Omega}{2 y(1-c)^{2}}\right] \hat{\psi}=0 .
$$

\section{LOCAL ANALYSIS}

\section{A. Temporal stability}

The stability equation (16) is now studied in the temporal framework; $k$ is given, real, and complex eigenfrequencies $\omega$ are looked for. Since viscosity has been set to zero, the system is conservative and can only present purely real eigenfrequencies or complex conjugate pairs. Since we focus on flows that are locally stable to axisymmetric disturbances, this implies that $\omega$ can only be real. Solely neutral temporal waves are therefore present in the flow.

In the case of swirling flows with uniform axial flow, the dispersion relation reduces to the one pertaining to a pure vortex with an added Doppler effect according to

$$
\omega=k \pm \sqrt{\Omega} \omega_{0, n},
$$

where $\omega_{0, n}$ are the axisymmetric Kelvin modes associated to the vortex without axial flow of circulation profile $K_{0}(y)$, each mode being labeled according to the number $n$ of zeroes of its eigenfunction. The reader is referred to the book of Saffman ${ }^{18}$ for details. Typical Kelvin modes of a pure vortex are displayed in Fig. 2, here in the case of the vortex in solid body rotation. The first branch $\omega_{0,1}$ is the outermost and fastest branch, characterized by the largest group velocity

$$
v_{g}=\frac{\partial \omega_{0,1}}{\partial k}
$$

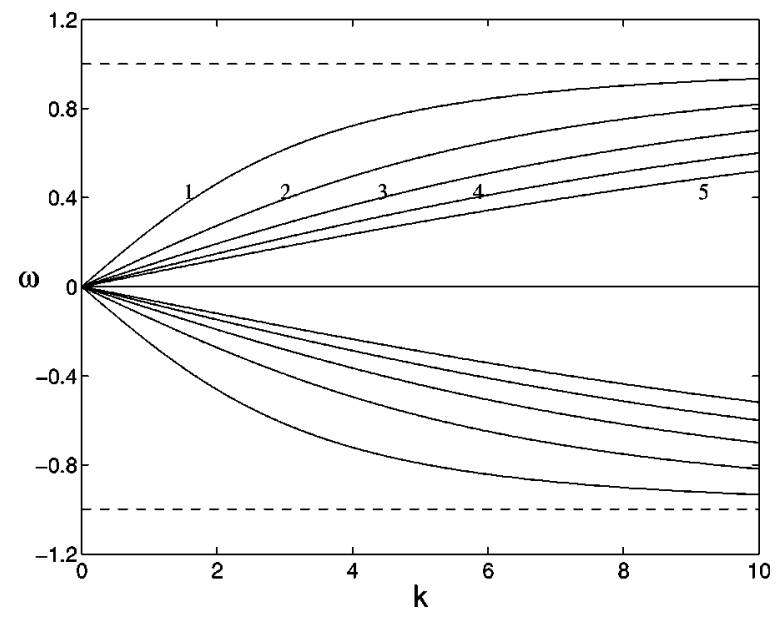

FIG. 2. Axisymmetric Kelvin waves sustained by a vortex column in solid body rotation of unit rotation rate without axial flow; different branches are labeled by an index $n$ indicating the number of zeroes of the corresponding eigenfunction. Symmetric negative $\omega$ branches have been added to the positive ones to ease the comparison with forthcoming cases where the axial velocity is nonzero.

with an overall maximum reached at $k=0$.

In the particular case of solid body rotation, the eigenfunctions as well as the dispersion relation may be expressed explicitly. On a given branch, it is found that the eigenfunctions do not depend on $k$ and take the form

$$
f_{n}(y)=\sqrt{y} J_{1}\left(b_{1 n} \sqrt{2 y}\right),
$$

for $n=1 \cdots \infty$, where $J_{1}$ is the Bessel function of order unity and $b_{1 n}$ its $n$th zero. In turn, Eq. (16) leads to the polynomial dispersion relation

$$
\left(k^{2}+b_{1 n}^{2}\right)(\omega-k)^{2}-\Omega k^{2}=0 .
$$

In the small wave number limit, expression (20) yields

$$
\omega \sim\left(1 \pm \frac{\sqrt{\Omega}}{b_{1, n}}\right) k
$$

This expression reflects the two contributions to the group velocity: the Doppler effect and the Kelvin wave weighted by the swirl parameter. The solutions of dispersion relation (20) are displayed in Fig. 3, where only $n=1$ and $n=2$ are plotted.

Three different cases are distinguished according to the amount of swirl introduced, with respect to the value of $\Omega_{B}=b_{1,1}^{2}$.

(1) When $\Omega<\Omega_{B}$ [Fig. 3(a)], the Doppler effect is so strong that the frequency $\omega$ is positive for all $k$. The group velocity $v_{g}=\partial \omega / \partial k$ as well as the phase velocity $c=\omega / k$ are positive for all branches and all $k$. According to Benjamin, ${ }^{2}$ the flow is said to be supercritical since it only sustains waves of positive phase velocity. It is also noted that, as $k \rightarrow \infty, \omega \rightarrow k \pm \sqrt{\Omega}$, so that both the phase and group velocity tend to \pm 1 when $k$ becomes large.

(2) When $\Omega=\Omega_{B}$ [Fig. 3(b)], the group velocity as well as the phase velocity of the $n=1$ lower branch both vanish when $k \rightarrow 0$ but they remain positive for $k>0$. Let us reiterate with Leibovich ${ }^{36}$ that the phase velocity at $k=0$ is precisely the group velocity. The flow is said to be critical. 

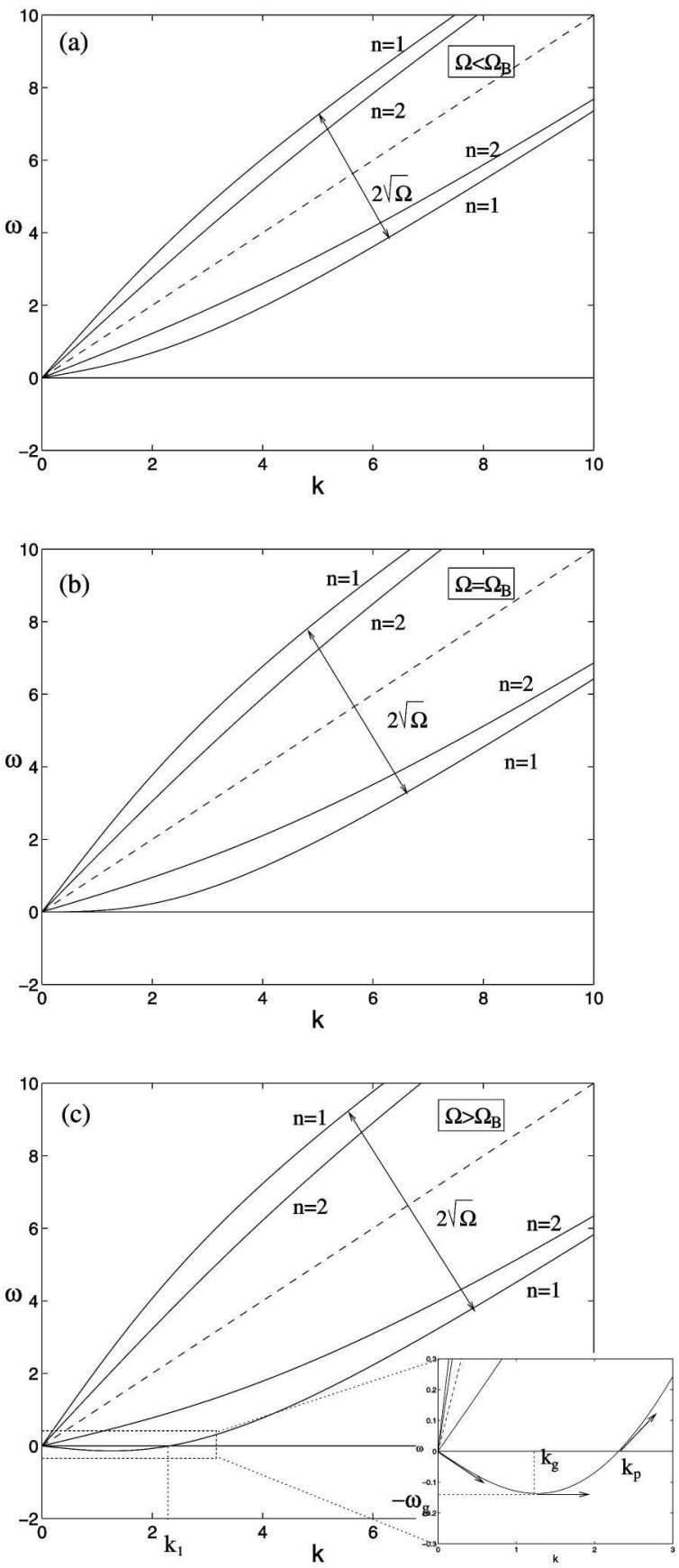

FIG. 3. Temporal stability. Frequency $\omega$ as a function of $k$ for the first two branches of Kelvin waves for different swirl settings: (a) $\Omega<\Omega_{B}$, (b) $\Omega=\Omega_{B}$, and (c) $\Omega>\Omega_{B}$.

(3) When $\Omega>\Omega_{B}$ [Fig. 3(c)], the group velocity as well as the phase velocity of the $n=1$ lower branch are negative when $k \rightarrow 0$. In reference to the negative sign of the phase velocity, the flow is said to be subcritical. The phase velocity remains negative in the wave-number range $0 \leqslant k \leqslant k_{p}$ which is wider than the wave-number range $0 \leqslant k \leqslant k_{g}$ where the group velocity is negative.

It is worth considering the general nature of the present discussion. For all stable azimuthal velocity profiles, the Kelvin branch structure is similar and the effect of adding a uniform axial flow follows along the lines above. If now we add a nonuniform axial flow with minimum $w_{\min }$ and maxi- mum $w_{\max }$, a forbidden cone appears defined by the two straight lines $\omega=w_{0}^{\min } k$ and $\omega=w_{0}^{\max } k$, as shown by Chandrasekhar ${ }^{37}$ or Leibovich. ${ }^{36}$ The reason lies in the existence of critical layers located at the radial position $y$ where the axial phase velocity equals the axial velocity of the base flow and the term $d \psi_{0} / d y-c$ in Eq. (16) vanishes. This point is discussed in great detail in Leibovich. ${ }^{36}$ The generic picture of Fig. 3 is nevertheless only slightly modified if we assume the minimum base flow axial velocity to be strictly positive $w_{0}^{\min }>0$. In that case, Leibovich ${ }^{36}$ has proven the existence of a finite nonzero critical swirl.

Squire ${ }^{1}$ was the first to interpret the value $\Omega_{B}$ as the critical swirl necessary to sustain a stationary wave in a medium of infinite extent. Since Benjamin ${ }^{2}$ subsequently introduced the super/subcritical concepts, the subscript $B$ is commonly used to designate Squire's critical swirl $\Omega_{B}$ and we will follow this convention. Squire ${ }^{1}$ also suggested that, as the swirl is increased above $\Omega_{B}$, stationary waves of finite wavelength exist and propagate disturbances upstream. This is the point that has been criticized by Benjamin ${ }^{2}$ on the grounds that the group velocity of the stationary wave of finite wave number $k_{p}$ is positive, as readily illustrated in Fig. 3(c) where the tangent to the Kelvin branch at $k_{p}$ is indeed positive. Figure 3 nevertheless shows that waves in the range $0 \leqslant k \leqslant k_{g}$ have negative group velocity and can transport energy upstream, although they correspond to $\omega$ $<0$ and are not stationary. As outlined in the Introduction, there has been quite a misunderstanding in the literature. Squire was looking for stationary waves because the bubble form of the vortex breakdown is observed to be a steady process. He had in mind an infinite domain and was considering waves with $\omega=0$. When the translational invariance is broken by inhomogeneities, as suggested by Leibovich, ${ }^{13}$ or by the consideration of inlet and outlet boundary conditions, an unstable global mode can be constructed as a superposition of traveling waves. A "static" instability may then develop with $\operatorname{Re}(\omega)=0$ as in the divergence or buckling of a loaded beam. This line of reasoning is expounded in Sec. V.

\section{B. Spatial stability}

In contrast to the temporal analysis, the spatial approach refers to the development in space of waves generated by a localized time-harmonic forcing. It is more appropriate for the analysis of the global instability of finite-length systems. A real frequency $\omega$ is prescribed and four spatial branches are found for each $n$ since the dispersion relation (20) admits four wave-number solutions.

Figures 4(b) and 4(e) display the four spatial branches in the $\omega-k_{r}$ plane for $n=1$. The purely real spatial branches correspond to the solutions already found in the temporal theory. This is readily seen in Figs. 4(a) and 4(d) deduced from the temporal waves of Fig. 3 by a simple $\pi / 2$ rotation of the axes $k-\omega$, in order to present them in the $\omega-k$ plane. We have also displayed the configurations of the branches in the negative $\omega$ half-plane by applying a simple symmetry. When the flow is supercritical $\left(\Omega<\Omega_{B}\right)$, only two real $k$ solutions exist [Fig. 4(a)], irrespective of the frequency $\omega$, whereas for subcritical flows $\left(\Omega>\Omega_{B}\right)$ four real 

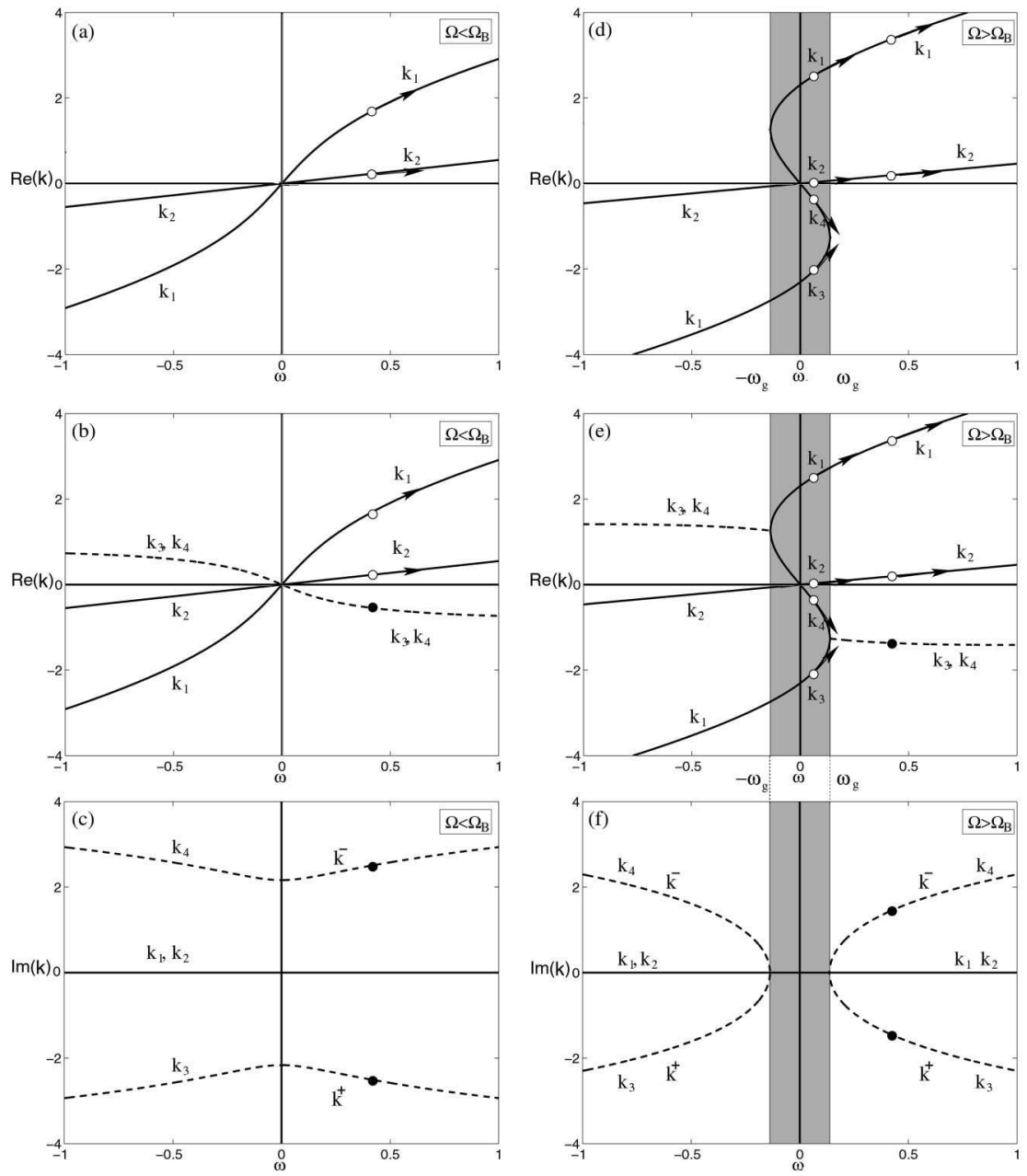

FIG. 4. Spatial stability. (a) ((d)): Representation of the real temporal waves of Fig. 3 for the first branch $n=1$ in the $\omega-k$ plane in the supercritical (subcritical) regime. (b) ((e)): Real part $\operatorname{Re}(k)$ of the spatial branches as a function of real frequency $\omega$ in the supercritical (subcritical) regime. (c) $((\mathrm{f}))$ : Corresponding imaginary part $\operatorname{Im}(k)$ as a function of $\omega$. Purely real wave numbers are denoted by solid lines and open circles. The sign of their group velocity is depicted by an arrow tangent to the curve, positive (negative) group velocities corresponding to upward (downward) pointing arrows. Complex wave numbers are denoted by dashed lines and closed circles. The direction of their propagation is then given by the sign of their imaginary part. The "neutral" range is highlighted in gray. spatial branches exist [Fig. 4(c)] in the frequency range $-\omega_{g} \leqslant \omega \leqslant \omega_{g}$. Only in that interval are the four spatial branches already captured by temporal theory. In the remaining part of the frequency domain and in the supercritical case one has to resort to the general local spatial stability theory in order to determine the remaining complex branches with $\operatorname{Im}(k) \neq 0$. They cannot be determined by this simple "rotation" of the temporal results.

Let us analyze the properties of spatial waves generated by forcing at a real frequency $\omega$. This is done by considering harmonic forcing of the form

$$
f(z, t)=\delta(z) H(t) e^{-i \omega t},
$$

where $H(t)$ is the Heaviside unit step function which models the starting of the forcing and thereby allows one to consider a causal response (see Huerre and Monkewitz ${ }^{38}$ for details). In a temporally stable system, it is well known that the response of the system to this harmonic forcing consists of a linear superposition of all possible spatial waves, each one corresponding to a root $k$ of the dispersion relation for $\omega$. These spatial waves separate into two groups, the $k^{+}$waves which travel downstream and the $k^{-}$waves which travel upstream. Once the direction of a spatial wave has been determined, it can be either evanescent or neutral but it cannot be amplified since the present conservative system is temporally neutral. For a neutral flow, and a given root $k(\omega)$ of the dispersion relation, its $k^{+}$or $k^{-}$nature is either determined by the corresponding real group velocity if $k(\omega)$ is real or directly by the sign of its imaginary part if $k(\omega)$ is complex (this is generally not the case for an unstable system where one has to resort to contour deformation arguments as discussed in Bers). ${ }^{39}$ In the case where $k(\omega)$ is complex, since there cannot exist spatially amplified waves, a wave with $\operatorname{Im}(k)<0$ necessarily propagates in the downstream direction and it is a $k^{-}$branch, whereas a spatial wave with $\operatorname{Im}(k)$ $>0$ necessarily propagates in the upstream direction and it is a $k^{+}$branch.

When the rigidly rotating column is considered, the dispersion relation (20) is polynomial in $k$ and there are always four spatial waves denoted $k_{1}(\omega), k_{2}(\omega), k_{3}(\omega)$, and $k_{4}(\omega)$. This last property is in fact general for other profiles once the dynamics has been projected onto the lowest radial eigenmode but the polynomial nature of the dispersion relation is lost. An interesting property of the system studied here is that, whatever real frequency is considered, there are always three $k^{+}$waves and one $k^{-}$wave. The three $k^{+}$ waves will be denoted by indices 1,2 , and 3 , the $k^{-}$wave by an index 4. It should be mentioned that this imbalance between the number of upstream and downstream waves is perfectly consistent with the prescribed boundary conditions (12). Three inlet conditions are imposed in accordance with 
the three downstream waves whereas only one outlet condition is imposed corresponding to one upstream propagating wave. This perfect matching explains the well-posedness of the problem with the selected boundary conditions.

Figures 4(b) and 4(c) [4(e) and 4(f)] depict the characteristic wave portrait in the supercritical case $\Omega<\Omega_{B}$ (in the subcritical case $\left.\Omega>\Omega_{B}\right)$. The transition from supercritical $\left(\Omega<\Omega_{B}\right)$ to subcritical $\left(\Omega>\Omega_{B}\right)$ corresponds to the following evolution in the topology of the spatial branches.

(1) When $\Omega<\Omega_{B}$, whatever real forcing frequency is applied, two waves $k_{1}$ and $k_{2}$ are neutral and propagate downstream as indicated by an arrow tangent to the branch in Fig. 4(b). The neutral branches $k_{1}$ and $k_{2}$ in that figure which are depicted by a full line and open circles, are exactly the same as those found in Fig. 4(a). Two other waves are complex conjugate (dashed line with a closed circle). Figure 4(c) depicts the imaginary part of the spatial branches: the downstream $k^{+}$evanescent wave $k_{3}$ has a negative imaginary part and conversely the upstream $k^{-}$evanescent wave $k_{4}$ has a positive imaginary part.

(2) When $\Omega>\Omega_{B}$, there exists a so-called static range of frequencies $-\omega_{g} \leqslant \omega \leqslant \omega_{g}$ where all four spatial waves are neutral. According to Doaré and de Langre, ${ }^{6}$ it is called static because it contains the frequency $\omega=0$. Outside of the "neutral" range, the branch portrait is similar as for $\Omega<\Omega_{B}$ and the reader is referred to the previous paragraph. In the neutral range, all four branches could have been deduced by the simple "rotation" from the temporal theory [compare Figs. 4(d) and 4(e)]. Three branches $k_{1}, k_{2}$, and $k_{3}$ have positive group velocities and propagate downstream whereas one branch $k_{4}$ has a negative group velocity and propagates upstream.

In neutrally stable flows, the transition from supercritical to subcritical, when considered from the spatial point of view, involves a pinching process similar to the one taking place in unstable flows for transition from convective to absolute instability. This feature is obvious if one compares the topology of Figs. 4(c) and 4(f).

\section{LINEAR GLOBAL MODES}

\section{A. Global analysis}

The point of view taken in the previous section was to consider the local properties of inertial waves for swirling flows in parallel infinite pipes. For a pipe of finite length, inlet and outlet conditions (12) must be added and a socalled global stability analysis must be performed. In this context Wang and Rusak ${ }^{15}$ have demonstrated that the global linear instability onset is equal to $\Omega_{1}=\Omega_{B}+\pi^{2} / 4 L^{2}$. This means that a necessary condition for flows to become globally unstable appears to be the local subcriticality of the flow. Moreover, when $L$ is increased to infinity, the global critical swirl $\Omega_{1}$ tends to $\Omega_{B}$. Such a finite box correction has also been discovered in the fully nonlinear calculations of Chomaz and Couairon. ${ }^{5}$

For fluid conveying elastic pipes, Doaré and de Langre ${ }^{6}$ have recently discovered that, in the long pipe limit, global instability arises as soon as all spatial waves become neutral in a particular range of forcing frequencies. In the same spirit as Doaré and de Langre, ${ }^{6}$ this section aims at elucidating the link between the local properties (neutral, evanescent) of the spatial waves and the existence of a global mode in swirling flows, via a consideration of the boundary conditions.

As already shown for the present swirling flow model by Wang and Rusak, ${ }^{15}$ perturbations are expanded on the basis of eigenfunctions (19) according to

$$
\begin{aligned}
& K^{\prime}(y, z, t)=\sum_{n=1}^{\infty} \widetilde{K}^{(n)}(z, t) f_{n}(y), \\
& \psi^{\prime}(y, z, t)=\sum_{n=1}^{\infty} \widetilde{\psi}^{(n)}(z, t) f_{n}(y),
\end{aligned}
$$

thereby yielding after substitution into (11) an infinite set of uncoupled one-dimensional partial differential equations for $\widetilde{K}^{(n)}$ and $\widetilde{\psi}^{(n)}$. Each equation corresponds to a particular Kelvin wave of index $n$. We consider here only the $n=1$ mode that first leads to destabilization and obeys the system

$$
\left[\begin{array}{cc}
1 & 0 \\
0 & \Omega_{B}-\frac{\partial^{2}}{\partial z^{2}}
\end{array}\right]\left(\begin{array}{c}
\dot{\tilde{K}} \\
\dot{\psi}
\end{array}\right)=\left[\begin{array}{cc}
-\frac{\partial}{\partial z} & \sqrt{\Omega} \frac{\partial}{\partial z} \\
\sqrt{\Omega} \frac{\partial}{\partial z} & \frac{\partial^{3}}{\partial z^{3}}-\Omega_{B} \frac{\partial}{\partial z}
\end{array}\right]\left(\begin{array}{c}
\widetilde{K} \\
\widetilde{\psi}
\end{array}\right),
$$

where $\widetilde{K}=\widetilde{K}^{(1)}$ and $\widetilde{\psi}=\widetilde{\psi}^{(1)}$ to ease the notation. The boundary conditions (12) read

$$
\widetilde{K}(0, t)=0 ; \quad \widetilde{\psi}(0, t)=0 ; \quad \widetilde{\psi}_{z z}(0, t)=0 ; \quad \widetilde{\psi}_{z}(L, t)=0 .
$$

In (24), the dot denotes the time derivative. In the spirit of Wang and Rusak, ${ }^{15}$ global eigenvalues and eigenfunctions of system (24)-(25) are now expressed in terms of a linear combination of Kelvin waves supported by the columnar base state. As noted in Sec. III B, for a given $\omega$, there are always four spatial branches $k_{1}(\omega), k_{2}(\omega), k_{3}(\omega)$, and $k_{4}(\omega)$ and we consider a combination of the form

$$
\begin{aligned}
& \widetilde{K}(z, t)=\sum_{i=1}^{4} A_{i} e^{i\left(k_{i} z-\omega t\right)}, \\
& \widetilde{\psi}(z, t)=\sum_{i=1}^{4} B_{i} e^{i\left(k_{i} z-\omega t\right)},
\end{aligned}
$$

where $A_{i}$ and $B_{i}$ are unknown complex amplitudes. Substitution into the governing equation (24) leads to the compatibility relation

$$
B_{i}=A_{i} \frac{k_{i}-\omega}{\sqrt{\Omega} k_{i}},
$$

and to the dispersion relation (20). Imposing the boundary conditions (25) to the linear combination (26) requires that the eigenfrequency $\omega$ satisfy the global eigencondition 


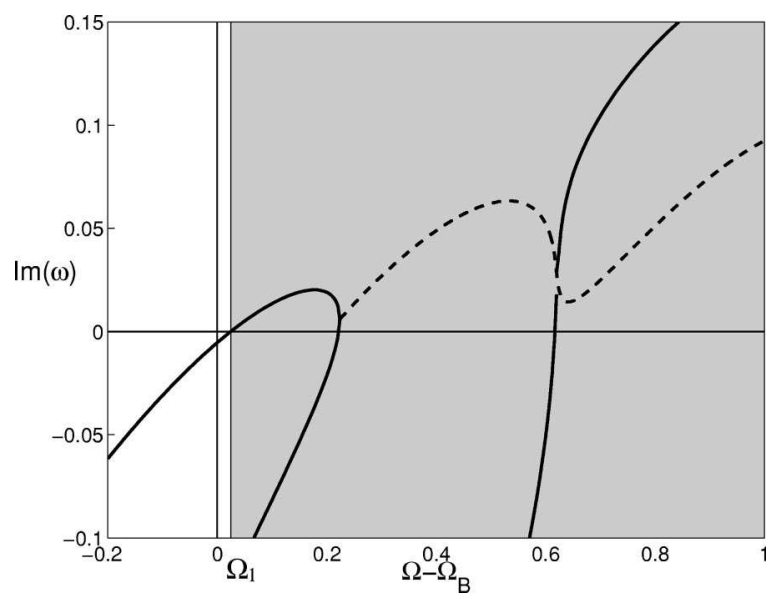

FIG. 5. Growth rate $\operatorname{Im}(\omega)$ of the most unstable global eigenvalues as a function of the swirl parameter $\Omega-\Omega_{B}$ for $L=10$. Eigenvalues with $\operatorname{Re}(\omega) \neq 0$ are displayed in dashed lines. The unstable region is shaded.

$$
D(\omega) \equiv\left|\begin{array}{cccc}
1 & 1 & 1 & 1 \\
\frac{\sqrt{\Omega} k_{1}}{k_{1}-\omega} & \frac{\sqrt{\Omega} k_{2}}{k_{2}-\omega} & \frac{\sqrt{\Omega} k_{3}}{k_{3}-\omega} & \frac{\sqrt{\Omega} k_{4}}{k_{4}-\omega} \\
k_{1}^{2} & k_{2}^{2} & k_{3}^{2} & k_{4}^{2} \\
k_{1} e^{i k_{1} L} & k_{2} e^{i k_{2} L} & k_{3} e^{i k_{3} L} & k_{4} e^{i k_{4} L}
\end{array}\right|=0 .
$$

The addition of boundary conditions to the dispersion relation (20) is seen to break the conservative nature of the system. Eigenfrequencies $\omega$ are not found in complex conjugate pairs but in anticomplex conjugate pairs, i.e., $\omega \leftrightarrow-\omega^{*}$. The leading eigenvalues are plotted in Fig. 5 as a function of the swirl parameter $\Omega-\Omega_{B}$. The first unstable mode effectively appears when $\Omega=\Omega_{1}=\Omega_{B}+\pi / 4 L^{2}$. At this threshold, the instability is static since the real part of its eigenfrequency $\operatorname{Re}(\omega)$ is zero. It is seen that several global modes become unstable in sequence as the swirl number is further increased. When two static branches of global modes collide, they interact to yield oscillatory modes with nonzero frequency $\operatorname{Re}(\omega)$.

\section{B. Reflection coefficients}

The global dispersion relation (28) may be reinterpreted in terms of propagation and reflection of waves in the following manner. Consider the leading real global eigenfrequency at instability onset. Let us start at $z=L$ with an amplitude $A_{4}$ of the upstream propagating wave; it reaches $z$ $=0$ with the amplitude $A_{4}^{\prime}=\mathbf{P}^{-} A_{4}$, where the $1 \times 1$ upstream propagation matrix $\mathbf{P}^{-}$equals

$$
\mathbf{P}^{-}=e^{-i k_{4} L} .
$$

The three-component vector of amplitudes $\left(A_{1}, A_{2}, A_{3}\right)^{T}$ that characterizes the reflected waves at the inlet $z=0$ is found by imposing the three inlet conditions in (25). In matrix form, this can be written $\left(A_{1}, A_{2}, A_{3}\right)^{T}=\mathbf{R}^{-} A_{4}^{\prime}$, where the $3 \times 1$ upstream reflection matrix $\mathbf{R}^{-}$is given by

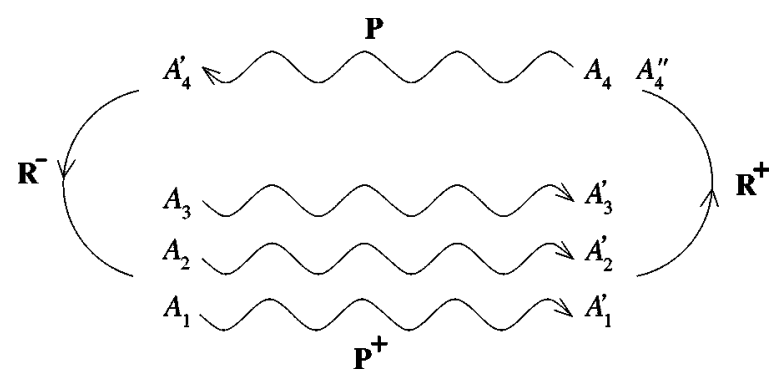

FIG. 6. Definition of upstream and downstream propagation and reflection matrices $\mathbf{R}^{+}, \mathbf{P}^{+}, \mathbf{R}^{-}$, and $\mathbf{P}^{-}$applied to the amplitudes $A_{1}, A_{2}, A_{3}$, and $A_{4}$.

$$
\mathbf{R}^{-}=-\frac{1}{f_{1,2}+f_{1,3}+f_{3,1}}\left[\begin{array}{l}
f_{2,3}+f_{3,4}+f_{4,2} \\
f_{1,4}+f_{4,3}+f_{3,1} \\
f_{1,2}+f_{2,4}+f_{4,1}
\end{array}\right] .
$$

In Eq. (30) the auxiliary functions $f_{i, j}$ have been introduced and they are defined as

$$
f_{i, j}=-\frac{\left(k_{i}-\omega\right)\left(k_{j}-\omega\right)\left(k_{i}-k_{j}\right)\left(k_{i}+k_{j}\right)}{\Omega k_{i} k_{j}} .
$$

The three waves then travel downstream and reach $x=L$ with amplitudes $A_{1} e^{i k_{1} L}, A_{2} e^{i k_{2} L}$, and $A_{3} e^{i k_{3} L}$, respectively. The downstream propagation may be expressed in matrix form, according to $\left(A_{1}^{\prime}, A_{2}^{\prime}, A_{3}^{\prime}\right)^{T}=\mathbf{P}^{+}\left(A_{1}, A_{2}, A_{3}\right)^{T}$, where the $3 \times 3$ propagation matrix $\mathbf{P}^{+}$is defined by

$$
\mathbf{P}^{+}=\left(\begin{array}{ccc}
e^{i k_{1} L} & 0 & 0 \\
0 & e^{i k_{2} L} & 0 \\
0 & 0 & e^{i k_{3} L}
\end{array}\right)
$$

Finally, the outlet reflection condition gives $A_{4}^{\prime \prime}$ $=\mathbf{R}^{+}\left(A_{1}^{\prime}, A_{2}^{\prime}, A_{3}^{\prime}\right)^{T}$, where the $1 \times 3$ downstream reflection matrix $\mathbf{R}^{+}$is defined by

$$
\mathbf{R}^{+}=-\frac{1}{k_{4}-\omega}\left(k_{1}-\omega, k_{2}-\omega, k_{3}-\omega\right) .
$$

The different propagation and reflection paths are sketched in Fig. 6.

At the global instability threshold, the amplitude of the upstream wave $A_{4}^{\prime \prime}$, after having completed one roundtrip, should be equal to $A_{4}$. This phase and energy closure principle reads in matrix form

$$
\mathbf{R}^{+} \mathbf{P}^{+} \mathbf{R}^{-} \mathbf{P}^{-}=\mathbf{1} .
$$

After a lot of strenuous algebra, it can be shown that the closure principle (34) is equivalent to the eigenrelation (28). The present formulation clearly isolates the role of the propagation in the bulk and of the reflection at the boundaries.

According to a general property of induced matrix norms ${ }^{40}$ the norm of a product of matrices is inferior or equal to the product of the norms. A necessary condition for Eq. (34) to be satisfied is therefore

$$
1 \leqslant\left\|\mathbf{P}^{-1}\right\|_{2}\left\|\mathbf{P}^{+}\right\|_{2}\left\|\mathbf{R}^{-}\right\|_{2}\left\|\mathbf{R}^{+}\right\|_{2} .
$$


Introducing $\mathcal{R}^{-}=\left\|\mathbf{R}^{-}\right\|_{2}$ and $\mathcal{R}^{+}=\left\|\mathbf{R}^{+}\right\|_{2}$ and taking into account the fact that the two-norm of a diagonal matrix equals the maximum of the modulus of its diagonal terms, the necessary condition (35) translates into

$$
1 \leqslant \exp \left[\left\{\operatorname{Im}\left(k_{4}\right)-\max _{i=1,2,3}\left[\operatorname{Im}\left(k_{i}\right)\right]\right\} L\right] \mathcal{R}^{-} \mathcal{R}^{+},
$$

where we recall that $k_{4}$ is the wave number of the upstream $k^{-}$wave and $k_{1}, k_{2}$, and $k_{3}$ are the $k^{+}$downstream propagating waves. Since two of the downstream spatial waves are always neutral, inequality (36) amounts to

$$
1 \leqslant \exp \left[\left\{\operatorname{Im}\left(k_{4}\right)\right\} L\right] \mathcal{R}^{-} \mathcal{R}^{+} .
$$

Let us reiterate that a real forcing frequency has been prescribed and that we examine if the finite length pipe is able to sustain a periodic cycle, in which case the flow will have been shown to be at the instability threshold. It should be noticed that $\mathcal{R}^{-}$and $\mathcal{R}^{+}$depend only on the inlet and outlet conditions and not on the length of the pipe $L$. If $\Omega<\Omega_{B}$ (supercritical range), the upstream propagating wave is evanescent and the term $e^{\left\{\operatorname{Im}\left(k_{4}\right)\right\} L}$ decays exponentially to zero as $L$ goes to infinity and therefore inequality (37) cannot be fulfilled. The flow is thus globally stable. In contrast, when $\Omega>\Omega_{B}$ (subcritical range) and $-\omega_{g} \leqslant \omega \leqslant \omega_{g}, e^{\left\{\operatorname{Im}\left(k_{4}\right)\right\}_{L}}=1$ and the flow may become globally unstable if the boundary conditions are overreflecting. Inequality (37) in fact imposes that $\mathcal{R}^{-} \mathcal{R}^{+} \geqslant 1$. This reasoning demonstrates without solving the global dispersion relation (28) that the global instability threshold $\Omega_{1}$ is necessarily above the critical swirl $\Omega_{B}$ when $L$ goes to infinity. Furthermore, global instability requires that the boundary conditions provide energy to the perturbations. The next section is devoted to the identification of the amplification mechanism that takes place at the pipe inlet and outlet.

\section{ENERGY BUDGET}

The local kinetic energy functional $e_{c}(z, t)$ of the perturbations at a local station $z$ is now expressed as the integral along the radial direction

$$
\begin{aligned}
e_{c}(z, t)= & \int_{0}^{1}\left[u^{\prime 2}(r, z)+v^{\prime 2}(r, z)+w^{\prime 2}(r, z)\right] r d r \\
= & \int_{0}^{1 / 2}\left[u^{\prime 2}(y, z)+v^{\prime 2}(y, z)+w^{\prime 2}(y, z)\right] d y \\
= & \int_{0}^{1 / 2}\left[\left(\frac{\partial \widetilde{\psi}(z, t)}{\partial z,}\right)^{2} \frac{f_{1}^{2}(y)}{2 y}+\widetilde{K}^{2}(z, t) \frac{f_{1}^{2}(y)}{2 y}\right. \\
& \left.+\widetilde{\psi}^{2}(z, t)\left(\frac{\partial f_{1}}{\partial y}\right)^{2}\right] d y .
\end{aligned}
$$

Since the radial eigenfunction verifies

$$
\frac{\partial^{2} f_{1}}{\partial y^{2}}=-\frac{\Omega_{B}}{2 y} f_{1},
$$

and $f_{1}(0)=f_{1}(1 / 2)=0$, an integration by parts yields

$$
\int_{0}^{1 / 2}\left(\frac{\partial f_{1}}{\partial y}\right)^{2} d y=\int_{0}^{1 / 2} \Omega_{B} \frac{f_{1}^{2}(y)}{2 y} d y .
$$

Up to a multiplicative constant, the kinetic energy density (37) is therefore

$$
e_{c}(z, t) \propto \frac{1}{2}\left[\widetilde{K}^{2}(z, t)+\Omega_{B} \widetilde{\psi}^{2}(z, t)+\left(\frac{\partial \widetilde{\psi}}{\partial z}\right)^{2}\right] .
$$

Denote finally the total kinetic energy by

$$
E_{c}(t)=\int_{0}^{L} e_{c}(z, t) d z
$$

Let us now multiply Eq. (11) by $(K, \psi)$ according to

$$
\begin{aligned}
\left(\begin{array}{ll}
\tilde{K} & \widetilde{\psi}
\end{array}\right)\left[\begin{array}{cc}
1 & 0 \\
0 & \Omega_{B}-\frac{\partial^{2}}{\partial z^{2}}
\end{array}\right]\left(\begin{array}{c}
\dot{\tilde{K}} \\
\dot{\tilde{\psi}}
\end{array}\right) \\
\quad=\left(\begin{array}{ll}
\widetilde{K} & \widetilde{\psi}
\end{array}\right)\left[\begin{array}{cc}
-\frac{\partial}{\partial z} & \sqrt{\Omega} \frac{\partial}{\partial z} \\
\sqrt{\Omega} \frac{\partial}{\partial z} & \frac{\partial^{3}}{\partial z^{3}}-\Omega_{B} \frac{\partial}{\partial z}
\end{array}\right]\left(\begin{array}{c}
\widetilde{K} \\
\widetilde{\psi}
\end{array}\right),
\end{aligned}
$$

and further integrate between 0 and $L$. Repeated integrations by parts yield

$$
\begin{aligned}
\dot{E}_{c}= & {\left[-\frac{\widetilde{K}^{2}}{2}-\Omega_{B} \frac{\widetilde{\psi}^{2}}{2}-\frac{1}{2} \frac{\partial \widetilde{\psi}^{2}}{\partial z}+\sqrt{\Omega} \widetilde{K} \widetilde{\psi}+\widetilde{\psi} \frac{\partial^{2} \widetilde{\psi}}{\partial z^{2}}\right.} \\
& \left.+\widetilde{\psi}\left(\frac{\partial \dot{\tilde{\psi}}}{\partial z}\right)^{2}\right]_{0}^{L} .
\end{aligned}
$$

The total kinetic energy of the perturbation is seen to grow if the right-hand side of Eq. (44) is positive and decrease if it is negative. The total kinetic energy of the perturbations may vary only as a result of the boundary terms. The bulk of the flow conserves the energy of the perturbations, a property which reflects the locally neutral nature of the Kelvin waves in the bulk.

The first case in Table I evaluates the contributions of each of the six boundary terms on the right-hand side of Eq. (44) at each end of the pipe with the boundary conditions (25) of Wang and Rusak. ${ }^{14}$ Some contributions vanish (0 in the table), some have a constant sign $(\ominus$ or $\oplus$ in the table), whereas some other (? in the table) cannot be evaluated without resorting to the full eigenmode calculation. For the boundary conditions prescribed by Wang and Rusak, ${ }^{14}$ one term is positive at the inlet and two terms remain undetermined at the downstream end. The full modal analysis of Sec. IV is necessary in order to conclude.

Since we have proven in the last section that the boundaries are active in the energy budget driving the instability whereas the bulk is either a damping agent in the supercritical case $\left(\Omega<\Omega_{B}\right)$ or at most an honest waveguide in the subcritical case $\left(\Omega>\Omega_{B}\right)$, it is tempting to modify the boundary conditions and reexamine the global stability of the flow both in terms of a modal analysis, as in Sec. IV, and of the present energy budget analysis. 
TABLE I. Contribution of the different boundary terms in the energy growth equation (44) at each boundary point $z=0$ and $z=L$ for different sets of boundary conditions. The boundary conditions $\widetilde{\psi}(0)=0$ and $\widetilde{K}(0)=0$ that impose the inlet velocity profiles are maintained for all four cases. Reference case 1: boundary conditions of Wang and Rusak (Ref. 14); case 2: same as case 1 but with modified inlet boundary condition; case 3: same as case 1 but with modified outlet boundary condition; case 4: same as case 1 with two modified boundary conditions capable of quenching the global instability. Boundary terms are, respectively, designated by $0, \ominus, \oplus$, and ? according to whether they are, respectively, inactive, stabilizing, destabilizing, or a priori undetermined.

\begin{tabular}{|c|c|c|c|c|c|c|c|}
\hline Case & Boundary condition & $-\frac{\widetilde{K}^{2}}{2}$ & $-\Omega_{B} \frac{\widetilde{\psi}^{2}}{2}$ & $-\frac{1}{2}\left(\frac{\partial \widetilde{\psi}}{\partial z}\right)^{2}$ & $\sqrt{\Omega} \widetilde{K} \widetilde{\psi}$ & $\widetilde{\psi} \frac{\partial^{2} \tilde{\psi}}{\partial z^{2}}$ & $+\tilde{\psi} \frac{\partial \tilde{\psi}}{\partial z}$ \\
\hline \multirow[t]{2}{*}{1} & $\widetilde{\psi}_{z z}(0)=0$ & 0 & 0 & $\oplus$ & 0 & 0 & 0 \\
\hline & $\psi_{z}(L)=0$ & $\theta$ & $\theta$ & 0 & $?$ & $?$ & 0 \\
\hline \multirow[t]{2}{*}{2} & $\psi_{z}(0)=0$ & 0 & 0 & 0 & 0 & 0 & 0 \\
\hline & $\psi_{z}(L)=0$ & $\ominus$ & $\theta$ & 0 & $?$ & $?$ & 0 \\
\hline \multirow[t]{2}{*}{3} & $\psi_{z z}(0)=0$ & 0 & 0 & $\oplus$ & 0 & 0 & 0 \\
\hline & $\psi(L)=0$ & $\ominus$ & 0 & $\ominus$ & 0 & 0 & 0 \\
\hline \multirow[t]{2}{*}{4} & $\psi_{z}(0)=0$ & 0 & 0 & 0 & 0 & 0 & 0 \\
\hline & $\psi(L)=0$ & $\theta$ & 0 & $\theta$ & 0 & 0 & 0 \\
\hline
\end{tabular}

Wang and Rusak ${ }^{14}$ discuss the general nature of their boundary conditions. The boundary conditions $\widetilde{\psi}(z=0)=0$ and $\widetilde{K}(z=0)=0$ that impose the inlet velocity profiles are essential and maintained throughout the study. They argue that their analysis is not strongly dependent on the outlet condition (9), whereas the inlet condition (8) which lets the inlet radial velocity unprescribed is of highest importance to let the flow evolve towards vortex breakdown. We therefore define in addition to the reference case 1 of Wang and Rusak, ${ }^{14}$ three other test cases with modified boundary conditions. Case 2 has a strongly constrained inlet condition of vanishing radial velocity perturbation $\widetilde{\psi}_{z}(z=0)=0$ instead of $\widetilde{\psi}_{z z}(z=0)=0$. Case 3 has a more constrained outlet condition $\widetilde{\psi}(z=L)=0$ imposing no axial velocity perturbation instead of the zero radial velocity condition $\widetilde{\psi}_{z}(z=L)=0$. Finally case 4 cumulates both modifications of cases 2 and 3 . These modified boundary conditions are meant to let the mathematical problem be posed. They may be easily implemented in a numerical code and some of them have already been used (Brown and Lope ${ }^{28}$ prescribe, for instance, a vanishing radial inlet velocity perturbation as in our case 2 in their numerical treatment of the related viscous swirling flow in a pipe of finite length at $\mathrm{Re}=250$ ). The experimental feasibility of these modified boundary conditions sets (cases 2, 3 , and 4) is not the crucial issue here since they have been designed to analyze the energy budget of the flow. If all boundary conditions can be implemented individually, the complexity comes from imposing several at the same time. Zero inlet radial velocity perturbation $\widetilde{\psi}_{z}(z=0)=0$ (case 2) may be achieved for instance by the addition of a rotating honeycomb at the inlet. However, imposing an inlet azimuthal velocity different from solid body rotation would then require a complex experimental device ensuring a radius-depending differential rotation of the honeycomb.

Figure 7 presents the growth rate of the most unstable global modes in cases 2 and 3 (where they are identical) and case 4 to be compared with Fig. 5 for case 1. In Fig. 7(a) corresponding to cases 2 and 3 the global instability threshold is slightly increased to $\Omega_{1}^{(2)}=\Omega_{1}^{(3)}=\Omega_{B}+\pi^{2} / L^{2}$ to be compared with $\Omega_{1}^{(1)}=\Omega_{B}+\pi^{2} / 4 L^{2}$. Imposing zero perturbation radial velocity at the inlet or zero perturbation axial velocity at the outlet is therefore weakly stabilizing when compared with case 1 . Note, however, that the eigenfunctions along $z$ (not displayed) are different in cases 2 and 3 .
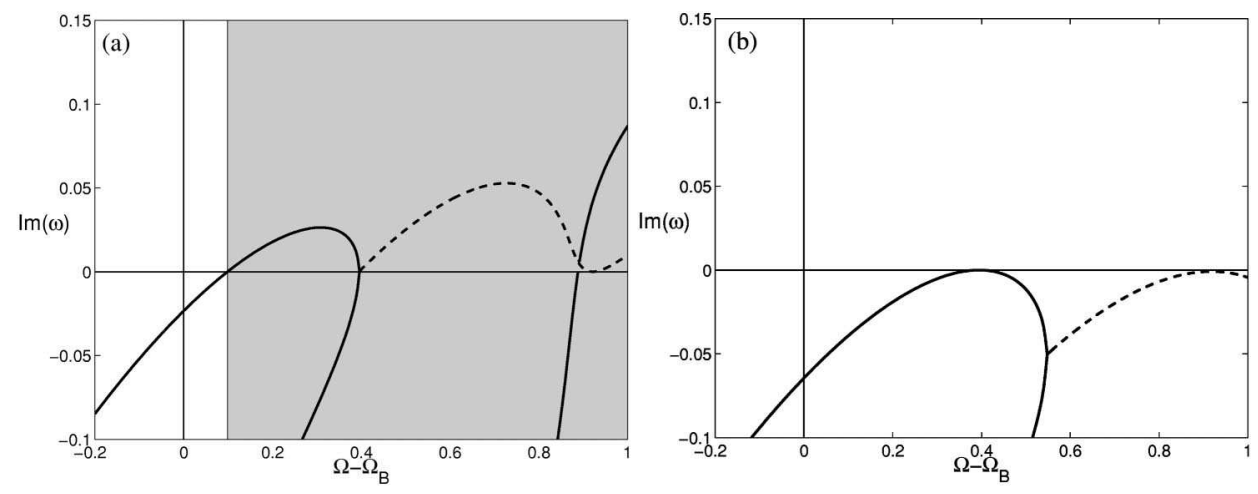

FIG. 7. Growth rate $\operatorname{Im}(\omega)$ of the most unstable global eigenvalues as a function of the swirl parameter $\Omega-\Omega_{B}$ for $L=10$. (a) Cases 2 and 3 where both curves are identical, and (b) case 4 . In comparison with Fig. 5, the global onset in cases 2 and 3 is delayed whereas case 4 is completely stabilized. 
The result for case 2 demonstrates that the degree of freedom of the inlet radial velocity is not a necessary condition for linear instability of the columnar state, contrary to the conjecture of Wang and Rusak. ${ }^{14}$ The nonlinear analysis of Wang and Rusak ${ }^{14}$ has, however, revealed the importance of this degree of freedom in order to let the flow evolve towards breakdown.

The most striking outcome is obtained in case 4 where both the upstream and downstream boundary conditions are changed. The flow is seen to remain stable, whatever value of the swirl is considered, as seen in Fig. 7(b). According to Table I, all of the 12 boundary terms vanish or are negative, thereby indicating that growth of the total kinetic energy is impossible. In cases 2 and 3, a precise examination of the boundary terms (see Table I) leads to the same conclusions as in case 1: a full modal analysis is required in order to determine the stable or unstable nature of the flow, as illustrated in Fig. 7(a). Note that in case 3 only the inlet term $1 /\left.2(\partial \widetilde{\psi} / \partial z)^{2}\right|_{0}$ is the only destabilizing agent whereas in case 2 only the outlet terms $\left.\sqrt{\Omega} \widetilde{K} \widetilde{\psi}\right|_{L}$ and $\left.\widetilde{\psi}\left(\partial^{2} \widetilde{\psi} / \partial z^{2}\right)\right|_{L}$ are destabilizing. The role of the inlet and outlet conditions is therefore subtle since both may have a destabilizing influence.

\section{CONCLUSION}

The main conclusions of the study may be summarized as follows. The swirling flow in a pipe model proposed by Wang and Rusak ${ }^{15}$ has been reexamined in light of local and global linear instability concepts. In the case of solid body rotation with uniform axial flow, the swirling flow is locally neutrally stable and supports traveling Kelvin waves. When suitable inlet and outlet end conditions are imposed, the flow nevertheless becomes globally unstable, which is sufficient to lead to incipient vortex breakdown. The transition to vortex breakdown does therefore not require the existence of a local instability mechanism induced either by axial shear, azimuthal shear, or centrifugal forces. According to Delbende, Chomaz, and Huerre ${ }^{10}$ or Yin et al. ${ }^{41}$ among others, the local instability features come into play to determine the unsteady helical structure which prevails in the wake of breakdown.

A spatial stability analysis in the bulk has been combined with a tracing analysis of the fate of the Kelvin waves as they bounce back and forth between the inlet and outlet boundaries. Through this detailed monitoring we have established a definite relationship between propagation in the bulk, reflection at the boundaries, and global instability. In this process, we have demonstrated that the local subcritical nature of the swirling flow is a necessary condition for global instability to arise.

An energy budget analysis has shown that the total perturbation energy can only grow through boundary contributions at the inlet and outlet. The boundary conditions selected by Wang and Rusak ${ }^{14}$ are indeed the most destabilizing, whereas other choices at the inlet and outlet lead to quenching of the global instability.

We have seen that the essential ingredient behind the global linear instability is the strong inhomogeneity introduced by the end conditions. It may also be asked whether a weak distributed axial inhomogeneity in the form of, e.g., slow pipe area variations is also capable of inducing a destabilization of Kelvin waves. Such a study would be in the same spirit as the asymptotic trapped wave analysis of Randall and Leibovich. ${ }^{42}$

\section{ACKNOWLEDGMENTS}

The authors wish to thank O. Doare for friendly, stimulating, and efficient discussions and P. Schmid and P. Huerre for helpful suggestions.

${ }^{1}$ H. B. Squire, "Analysis of the vortex breakdown phenomenon," Miszallaneen der Angewandten Mechanik (1960), pp. 306-312.

${ }^{2}$ T. B. Benjamin, "Theory of the vortex breakdown phenomenon," J. Fluid Mech. 14, 593 (1962).

${ }^{3} \mathrm{M}$. Gaster, "A note on the relation between temporally-increasing and spatially-increasing disturbances in hydrodynamic stability," J. Fluid Mech. 14, 222 (1962).

${ }^{4}$ P. Huerre and M. Rossi, "Hydrodynamic instabilities in open flows," in Hydrodynamic and Nonlinear Instabilities, edited by C. Godrèche and P. Manneville (Cambridge University Press, Cambridge, England, 1998), pp. 81-294.

${ }^{5}$ J.-M. Chomaz and A. Couairon, "Against the wind," Phys. Fluids 11, 2977 (1999).

${ }^{6}$ O. Doaré and E. De Langre, "Local and global stability of fluid conveying pipes on elastic foundations," J. Fluids Struct. 16, 1 (2002).

${ }^{7}$ Squire's standing waves might equally be called stationary waves.

${ }^{8}$ M. P. Escudier, "Vortex breakdown: Observations and explanations," Prog. Aerosp. Sci. 25, 189 (1988)

${ }^{9}$ C.-Y. Tsai and S. Widnall, "Examination of a group-velocity criterion for breakdown of a vortex flow in a divergent duct," Phys. Fluids 23, 864 (1980).

${ }^{10}$ I. Delbende, J.-M. Chomaz, and P. Huerre, “Absolute/convective instabilities in the Batchelor vortex: A numerical study of the linear impulse response," J. Fluid Mech. 355, 229 (1998).

${ }^{11}$ C. Olendraru, A. Sellier, and P. Huerre, "Inviscid instability of the Batchelor vortex: Absolute/convective transition and spatial branches," Phys. Fluids 11, 1805 (1999).

${ }^{12}$ T. Loiseleux, J.-M. Chomaz, and P. Huerre, "The effect of swirl on jets and wakes: Linear instability of the Rankine vortex with axial flow," Phys. Fluids 10, 1120 (1998).

${ }^{13}$ S. Leibovich, "Vortex stability and breakdown: Survey and extension," AIAA J. 22, 1192 (1984).

${ }^{14} \mathrm{~S}$. Wang and Z. Rusak, "The dynamics of a swirling flow in a pipe and transition to axisymmetric vortex breakdown," J. Fluid Mech. 340, 177 (1997).

${ }^{15} \mathrm{~S}$. Wang and Z. Rusak, "On the stability of an axisymmetric rotating flow in a pipe," Phys. Fluids 8, 1007 (1996)

${ }^{16}$ S. Wang and Z. Rusak, "On the stability of non-columnar swirling flows," Phys. Fluids 8, 1017 (1996).

${ }^{17}$ J. Keller, W. Egli, and W. Exley, "Force- and loss-free transitions between flow states," ZAMP 36, 854 (1985).

${ }^{18}$ P. G. Saffman, Vortex Dynamics (Cambridge University Press, Cambridge, England, 1992).

${ }^{19}$ L. Rayleigh, "On the dynamics of revolving fluids," Proc. R. Soc. London, Ser. A 93, 148 (1916).

${ }^{20}$ L. Howard and A. Gupta, "On the hydrodynamic and hydromagnetic stability of swirling flows," J. Fluid Mech. 14, 463 (1962).

${ }^{21}$ H. Ludwieg, "Stabilität der Strömung in einem zylindrischen Ringraum," Z. Flugwiss. 8, 135 (1960).

${ }^{22} \mathrm{~S}$. Leibovich and K. Stewartson, "A sufficient condition for the instability of columnar vortices," J. Fluid Mech. 126, 335 (1983).

${ }^{23} \mathrm{G}$. Batchelor and A. Gill, "Analysis of the stability of axisymmetric jets," J. Fluid Mech. 14, 529 (1964).

${ }^{24}$ Z. Rusak, S. Wang, and C. Whiting, "The evolution of a perturbed vortex in a pipe to axisymmetric vortex breakdown," J. Fluid Mech. 366, 211 (1998).

${ }^{25}$ S. Leibovich, "Weakly nonlinear waves in rotating fluids," J. Fluid Mech. 42, 803 (1970).

${ }^{26}$ A. Szeri and P. Holmes, "Nonlinear stability of axisymmetric swirling 
flows," Philos. Trans. R. Soc. London, Ser. A 326, 327 (1988).

${ }^{27} \mathrm{Z}$. Rusak, "The interaction of near-critical swirling flows in a pipe with inlet azimuthal vorticity perturbations," Phys. Fluids 10, 1672 (1998).

${ }^{28}$ G. Brown and J. Lopez, "Axisymmetric vortex breakdown. Part 2: Physical mechanisms," J. Fluid Mech. 221, 553 (1990).

${ }^{29}$ M. Hafez, G. Kuruvila, and M. D. Salas, "Numerical study of vortex breakdown," Appl. Num. Math. 2, 291 (1986).

${ }^{30} \mathrm{R}$. Kopecky and K. Torrance, "Initiation and structure of axisymmetric eddies in a rotating stream," Comput. Fluids 1, 289 (1973).

${ }^{31}$ E. Krause, "The solution to the problem of vortex breakdown," Lect. Notes Phys. 371, 35 (1984).

${ }^{32} \mathrm{D}$. Darmofal, "The role of vorticity dynamics in vortex breakdown," AIAA Pap. 93-3036 (1993).

${ }^{33}$ D. Snyder and R. E. Spall, "Numerical simulation of bubble-type vortex breakdown within a tube-and-vane apparatus," Phys. Fluids 12, 603 (2000).

${ }^{34} \mathrm{P}$. Beran and F. Culick, "The role of non-uniqueness in the development of vortex breakdown in tubes," J. Fluid Mech. 242, 491 (1992).
${ }^{35} \mathrm{~J}$. Lopez, "On the bifurcation structure of axisymmetric vortex breakdown in a constricted pipe," Phys. Fluids 6, 3683 (1994).

${ }^{36}$ S. Leibovich, "Waves in parallel or swirling stratified shear flows," J. Fluid Mech. 93, 401 (1979).

${ }^{37}$ S. Chandrasekhar, Hydrodynamic and Hydromagnetic Stability (Clarendon, Oxford, 1961).

${ }^{38}$ P. Huerre and P. A. Monkewitz, "Absolute and convective instabilities in free shear layers," J. Fluid Mech. 159, 151 (1985).

${ }^{39}$ A. Bers, "Space-time evolution of plasma instabilities-absolute and convective," in Handbook of Plasma Physics, edited by M. Rosenbluth and R. Sagdeev (North-Holland, Amsterdam, 1983), pp. 451-517.

${ }^{40}$ L. N. Trefethen and D. Bau, Numerical Linear Algebra (SIAM, Philadelphia, 1997).

${ }^{41}$ X. Yin, D. Sun, M. Wei, and J. Wu, "Absolute and convective instability character of slender viscous vortices," Phys. Fluids 12, 1062 (2000).

${ }^{42}$ J. D. Randall and S. Leibovich, "The critical state: A trapped wave model of vortex break-down," J. Fluid Mech. 58, 495 (1973). 